\title{
Pharmacokinetics of bupivacaïne in combination with clonidine versus dexmedetomidine in spinal anesthesia
}

\author{
H. mehdi ${ }^{1}$, E. Moez ${ }^{1}$, B. Ines ${ }^{1}$, N. Atouani ${ }^{1}$, A. \\ Eddhif ${ }^{1}$, C. Ghouibi ${ }^{1}$ \\ ${ }^{1}$ trauma center - tunis (Tunisia)
}

\section{Background and Goal of Study:}

Comparison of the plasma pharmacokinetics of bupivacaine in combination with clonidine versus dexmedetomidine in a spinal anesthesia.

Materials and Methods: randomized, double-blind prospective study including 40 patients ASA I or II, admitted for surgery for fracture of the femoral neck under spinal

anesthesia. divided into 2 groups: GC

(injection of $10 \mathrm{mg}$ of bupivacaine hyperbare $0,5 \%+15 \mu \mathrm{g}$ clonidine) and GD

(10 mg hyperbaric bupivacaine $+3 \mu \mathrm{g}$ dexmedetomidine) neither the patient nor the pharmacologist know the nature of the adjuvant.another anesthesiologist who assured only transport of samples, evaluates the time and duration of sensory block (prick-touch test at T12) and motor

(modified Bromage score> 2) Venous blood samples of $3 \mathrm{ml}$ were obtained before bupivacaine injection and immediately after spinal injection and at 5 , 10, 30, 45, 60, 120 and $240 \mathrm{~min}$ after the final injection. The plasma separated by centrifugation of blood samples and stored at $-20^{\circ} \mathrm{C}$ until use. Bupivacaine quantification in samples was carried out using HPLC method with ultraviolet absorbance detection $(\lambda=207 \mathrm{~nm})$.

Statistic study with SPSS22.

\section{Results and Discussion:}

\begin{tabular}{|c|c|c|c|}
\hline & $\overline{G C}$ & GD & p \\
\hline delay sensitif block (min) & $7,8 \pm 2,6$ & $5,7 \pm 1,9$ & 0,044 \\
\hline delay motor block (min) & $10,8 \pm 3,4$ & $10,213,1$ & 0,21 \\
\hline duration of at (min) & $54,6 \pm 16,2$ & $50,8 \pm 19,1$ & 0,54 \\
\hline duration of motor bloc(min) & $92,2 \pm 31,6$ & $101,1 \pm 29,4$ & 0,07 \\
\hline duration of sensitit bloc (min) & $151,4 \pm 39,2$ & $176,6 \pm 44,6$ & 0,031 \\
\hline Systemic dlairance Cl(m//min) & $930 \pm 282$ & $818 \pm 131$ & 0,024 \\
\hline Volume of distribution VD (I) & $102 \pm 24,8$ & $118 \pm 41,8$ & 0,067 \\
\hline Plasmatic halt-lite $T 1 / 2$ (min) & $88,7 \pm 34,9$ & $104,6 \pm 49,6$ & 0,029 \\
\hline $\begin{array}{l}\text { Maximal plasma bupivacaine } \\
\text { concentration Cmax ( }(\nu 9 / \mathrm{min})\end{array}$ & $0,668 \pm 0,234$ & $0,412 \pm 0,186$ & 0,034 \\
\hline $\begin{array}{l}\text { Time to reach the peak plasmlla } \\
\text { concentration Tmax (min) }\end{array}$ & $38,66 \pm 16,44$ & $51,29 \pm 18,87$ & 0,041 \\
\hline
\end{tabular}

Conclusion: Dexmedetomidine decreases the systemic absorption of bupivacaine compared to clonidine during spinal anesthesia, which could explain the extension of sensory block. 